\title{
Expanding peer review
}

\section{What can be done to reduce the burden on scientific referees while ensuring the continuity and quality of peer review?}

Peer review, in which scientists critically evaluate ideas, results and scientific models, is central to all scientific discourse and especially critical for ensuring the quality of the scientific literature. Researchers profit from the peer review process in their roles as authors, where it improves their published papers. They also benefit as referees by getting a broad view of leading studies in their field and by enhancing the rigor of their discipline's published literature. Yet, in the current climate of greater scientific output, peer review is just one component of the expanding workload of scientists. Last month (Nat. Chem. Biol. 6, 245, 2010) we answered some frequently asked questions about the peer review process at Nature Chemical Biology. This month we suggest ways to broaden the voices participating in peer review and reduce its demands on individual scientists.

As technological advances have equipped scientists with new tools to probe scientific questions in unprecedented ways, the pace of research has expanded significantly, particularly in interdisciplinary areas. This more competitive landscape has placed increasing pressure on scientists to publish their research in leading journals. More manuscripts are being written and a higher burden for each manuscript to include more and better data puts corresponding pressure on the peer review system. Unfortunately, this is happening in an environment where the 'to do' lists of scientists are already becoming unmanageable.

Despite everyone's best efforts, the slowest step in the publication process remains the evaluation of manuscripts by anonymous experts. All journals, including Nature Chemical Biology, strive to balance the desires of authors for expeditious review with our need for the high quality referee feedback necessary for making informed editorial decisions. Given these competing demands, the scientific community needs to find ways to reduce the burden of peer review, while making sure that it fulfills its central role in the advancement of science.

Journals can do their part by guaranteeing that peer review responsibilities are well distributed across their communities. At Nature Chemical Biology, we limit how frequently individual scientists are contacted to serve as referees, which helps to manage their reviewing load and balance their scientific influence at the journal. Because each journal is editorially independent, editors are unaware whether a scientist is already reviewing for another journal at Nature Publishing Group or elsewhere. Thus, we rely on referees to assess their review commitments and accept only those commissions for which they can devote the necessary attention to the paper in the allotted time period.

Scientists must also contribute to expanding the refereeing base of their communities. At a minimum, active researchers should review for journals that they regularly read, submit to or publish in with a frequency that takes into account that each paper they submit will be reviewed by, on average, three individuals. Scientists who are not reviewing as frequently as they might like should take a more active role by contacting editors and volunteering their time. These small steps by individual scientists will help, but a broader strategy is needed to support scientific peer review in the future.

From our side, Nature Chemical Biology is committed to expanding our referee database to ensure that our referee pool reflects the scientific, demographic and geographical diversity of our authors and readers. In adding scientists to our database of potential reviewers, the editors rely on our familiarity with the different fields of chemical biology, as well as contacts made at conferences or lab visits. Authors consistently provide excellent referee suggestions, and scientists who decline referee requests frequently suggest appropriate alternates. We also use online resources and search engines to identify potential referees, but this mechanism requires that scientists be 'findable' on the Internet. Thus, we encourage scientists, universities and companies to consider this as they design and update their websites.

Developing better systems to train young scientists in the peer review process is another main component of this strategy. Current curricula have been successful in developing the analytical thinking skills of students. For example, research group meetings and seminar courses frequently include student presentations that discuss and critique published papers. Yet these skills are just one component of rigorous peer review. We urge principal investigators to work with their colleagues and institutions to establish formal peer review training in their curricula, either as a part of existing courses or as separate modules. Such programs should focus on the intellectual aspects of review, such as how to assess the aims and technical merit of scientific studies. However, they also need to include training on the practical matters of how to express constructive criticism clearly in writing and should examine the professional and ethical dimensions of peer review. Such approaches will help young scientists develop peer reviewing skills, which will also shape students' views of how to design and evaluate their own scientific work.

As with most educational activities, there is no substitute for 'learning by doing'. Scientists and journals should facilitate referee training more broadly. Referees at Nature Chemical Biology are generally academic faculty, institute members or scientists in industry. Occasionally these referees will ask whether they may review manuscripts with colleagues, including members of their laboratories. We welcome this approach, provided that the principal investigator includes only one co-referee, communicates the contributor's identity to the editors and ensures that the extra participant agrees to keep the manuscript confidential. The responsibility for the quality of the final report remains with the commissioned referee, but, in our experience, collaborative review involving senior scientists and their junior colleagues results in more comprehensive and useful comments while providing an outstanding training opportunity. After review, we routinely send anonymous copies of all referee reports to the referees of each manuscript; we encourage all referees to examine these and discuss them with any collaborating coreferees, as the combined reports provide a useful way to view the review process more comprehensively and learn from the comments of other scientists.

Taken together, the contributions of individual referees as well as training programs in peer review will expand the peer review network to incorporate the broadest base of referees necessary for supporting the expanding scientific community and literature. 